\title{
Implication of IL-2/IL-21 region in systemic sclerosis genetic susceptibility
}

Lina-Marcela Diaz-Gallo, ${ }^{1}$ Carmen P Simeon, ${ }^{2}$ Jasper C Broen, ${ }^{3}$ Norberto Ortego-Centeno, ${ }^{4}$ Lorenzo Beretta, ${ }^{5}$ Madelon C Vonk, ${ }^{3}$ Patricia E Carreira, ${ }^{6}$ Sofia Vargas, ${ }^{1}$ José Andrés Román-Ivorra, ${ }^{7}$ Miguel A González-Gay, ${ }^{8}$ Carlos Tolosa, ${ }^{9}$ Francisco Javier López-Longo, ${ }^{10}$ Gerard Espinosa, ${ }^{11}$ Esther F Vicente, ${ }^{12}$ Roger Hesselstrand, ${ }^{13}$ Gabriela Riemekasten, ${ }^{14}$ Torsten Witte, ${ }^{15}$ Jörg H W Distler, ${ }^{16}$ Alexandre E Voskuyl, ${ }^{17}$ Annemie J Schuerwegh, ${ }^{18}$ Paul G Shiels, ${ }^{19}$ Annika Nordin, ${ }^{20}$ Leonid Padyukov, ${ }^{20}$ Anna-Maria Hoffmann-Vold, ${ }^{21}$ Raffaella Scorza, ${ }^{5}$ Claudio Lunardi, ${ }^{22}$ Paolo Airo, ${ }^{23}$ Jacob M van Laar, ${ }^{24}$ Nicolas Hunzelmann, ${ }^{25}$ Birgit S Gathof, ${ }^{26}$ Alexander Kreuter, ${ }^{27}$ Ariane Herrick, ${ }^{28}$ Jane Worthington, ${ }^{28}$ Christopher $\mathrm{P}$ Denton, ${ }^{29}$ Xiaodong Zhou, ${ }^{30}$ Frank C Arnett, ${ }^{30}$ Carmen Fonseca, ${ }^{29}$ Bobby PC Koeleman, ${ }^{31}$ Shervin Assasi, ${ }^{30}$ Timothy R D J Radstake, ${ }^{32}$ Maureen D Mayes, ${ }^{30}$ Javier Martín, ${ }^{1}$ The Spanish Scleroderma Group

- Additional supplementary data are published online only. To view these files please visit the journal online (http://dx.doi org/10.1136/annrheumdis2012-202357).

For numbered affiliations see end of article.

\section{Correspondence to Lina-Marcela Diaz-Gallo, Cellular Biology and Immunology Department, Instituto de Parasitología y Biomedicina López-Neyra (IPBLN-CSIC), Parque Tecnológico Ciencias de la Salud, Avenida del Conocimiento $\mathrm{s} / \mathrm{n}$ 18100-Armilla, Granada 18100, Spain; \\ lina.diaz@ipb.csic.es}

Accepted 21 October 2012 Published Online First 21 November 2012

\section{ABSTRACT}

Objective The interleukin 2 (/L-2) and interleukin 21 (IL-21) locus at chromosome 4q27 has been associated with several autoimmune diseases, and both genes are related to immune system functions. The aim of this study was to evaluate the role of the IL-2/IL-21 locus in systemic sclerosis (SSc).

Patients and methods The case control study included 4493 SSc Caucasian patients and 5856 healthy controls from eight Caucasian populations (Spain, Germany, The Netherlands, USA, Italy, Sweden, UK and Norway). Four single nucleotide polymorphisms (rs2069762, rs6822844, rs6835457 and rs907715) were genotyped using TaqMan allelic discrimination assays. Results We observed evidence of association of the rs6822844 and rs907715 variants with global SSc ( $p_{c}=6.6 E-4$ and $p_{c}=7.2 E-3$, respectively). Similar statistically significant associations were observed for the limited cutaneous form of the disease. The conditional regression analysis suggested that the most likely genetic variation responsible for the association was the rs6822844 polymorphism. Consistently, the rs2069762Ars6822844T-rs6835457G-rs907715T allelic combination showed evidence of association with SSc and limited cutaneous SSc subtype $\left(p_{c}=1.7 E-03\right.$ and $p_{c}=8 E-4$, respectively).

Conclusions These results suggested that the IL-2/IL-21 locus influences the genetic susceptibility to SSc. Moreover, this study provided further support for the IL-2/IL-21 locus as a common genetic factor in autoimmune diseases.

\section{INTRODUCTION}

Interleukin 2 (IL-2) and interleukin 21 (IL-21) are equally attractive biological candidates that may influence the pathogenesis of autoimmune diseases. Both are cytokines involved in the proliferation of
$\mathrm{T}$ and $\mathrm{B}$ lymphocytes and different immunological activation pathways. ${ }^{1}$ Moreover, the $I L-2$ and $I L-21$ genes cover a region of approximately $200 \mathrm{~kb}$ that maps in the 4q27 locus. IL-2 has an important role in the maintenance of immune system homeostasis and self-tolerance. This cytokine has two paradoxical roles: promoting $\mathrm{T}$ cell proliferation and terminating T cell responses. Moreover, IL-2 facilitates the production of immunoglobulins through B cells and induces the differentiation and proliferation of natural killer cells. ${ }^{12}$ IL-21 is a potent immunomodulatory cytokine with pleiotropic effects on both innate and adaptive immune responses. These actions include the following positive effects: enhanced proliferation of lymphoid cells, increased cytotoxicity of CD8 T cells and natural killer cells, and differentiation of $B$ cells into plasma cells. $I L-21$ is also produced by $\mathrm{T}$ helper 17 (Th17) cells and is a critical regulator of Th17 development. ${ }^{1}{ }^{3}$ Genetic association studies have demonstrated that several IL-2/IL-21 polymorphisms influence the risk for autoimmune diseases (AIDs). The first evidence of this association was found in type 1 diabetes, Graves' disease, coeliac diseases and rheumatoid arthritis. ${ }^{47}$ These results have been confirmed through replication studies in different populations and extended to other autoimmune diseases, such as inflammatory bowel diseases, giant cell arthritis, psoriasis and systemic lupus erythematosus (SLE). ${ }^{8-17}$

Systemic sclerosis (SSc) is a chronic fibrotic autoimmune disease in which patients are commonly classified into the following two major subgroups that are related to the specific autoantibodies against several nuclear and/or nucleolar antigens: (i) limited cutaneous SSc (lcSSc), which is related to the positive status of anticentromere autoantibodies (ACA) and (ii) diffuse cutaneous ( $\mathrm{dcSSc}$ ), which is related to the positive status of antitopoisomerase 
autoantibodies (ATA). ${ }^{18-22}$ More than 40 susceptibility loci to SSc have been identified during the last 10 years. Half of these variants need to be replicated in different populations and many of these variants are shared among different AIDs, especially SLE. ${ }^{22-25}$ In this regard, one single nucleotide polymorphism (SNP) of the $I L-2$ gene was proposed as risk factor to lcSSc subtype, ${ }^{26}$ but this association has not been confirmed by other studies. Moreover the IL-21 gene has been implicated as a potential driver of AIDs and recently a fine-mapping in SLE demonstrated that variants of the IL-2/IL-21 region are implicated in the genetic susceptibility to SLE. ${ }^{12} 16$ Thus, the aim of this study was to evaluate the influence of the $I L-2 / I L-21$ region in SSc genetic susceptibility.

\section{PATIENTS AND METHODS}

\section{Subjects}

This case-control association study was comprised of 4493 SSc patients and 5896 controls of Caucasian ancestry. The discover cohort included the Spanish group, which consisted of 1176 SSc patients and 1721 healthy controls. The follow-up phase consisted of the following subjects: 609 SSc cases and 426 controls from Germany, 365 SSc cases and 734 controls from the Netherlands, 916 SSc cases and 884 controls from USA, 595 SSc cases and 1107 controls from Italy, 225 SSc cases and 273 controls from Sweden, 374 SSc cases and 436 controls from the UK and 102 SSc cases and 278 controls from Norway. There was an overlapping of 1726 SSc and 2578 controls with the previous GWAS in SSc. ${ }^{25}$ The patients fulfilled the 1980 American College of Rheumatology classification criteria for $\mathrm{SSc}^{27}$ or the criteria proposed for early SSc. ${ }^{21}$ In addition, the patients were classified as having lcSSc or dcSSc as described by LeRoy et al. ${ }^{21}$ The following clinical data were collected for the ascertainment of the clinical phenotype of the SSc patients: age, gender and presence of SSc-specific autoantibodies (Ab; ACA and ATA). The control population consisted of unrelated healthy individuals recruited in the same geographical regions as the SSc patients, and they were matched by age, sex and ethnicity with the SSc patient groups. The study was approved by local ethical committees from all the participating centres. Both patients and controls were included in the study after written informed consent was obtained.

\section{SNP Selection and genotyping}

Four SNPs of the $I L-2 / I L-21$ region were selected for this study. The rs2069762 SNP was selected because it has been suggested to be a genetic factor of IcSSc subtype susceptibility by a study in a small Italian cohort. ${ }^{26}$ SSc and SLE share some immunogenetic pathways; thus, the rs6822844, rs6835457 and rs907715 IL-2/IL-21 polymorphisms were studied because they are the most associated variants in a recent fine-mapping of the region in SLE. ${ }^{12}$

DNA from the patients and the controls were extracted from peripheral white blood cells following standard procedures. The samples were genotyped for the rs2069762, rs6822844, rs6835457 and rs907715 IL-2/IL-21 region polymorphisms using predesigned SNP genotyping assays from Applied Biosystems (Assay IDs: C_15859930_10, C_28983601_10, C_1597475_10 and C__ 8949748_10, respectively). TaquMan SNP genotyping was performed using a 7900HT Real-Time PCR system from Applied Biosystems following the manufacturer's suggestions (Foster City, California, USA). In all the cohorts, the genotyping success rate was greater than $95 \%$, and randomly selected samples were genotyped twice to verify the genotyping accuracy. Ninety-nine per cent of the genotypes were identical.

\section{Statistical analysis}

The Hardy-Weinberg equilibrium was tested for all the SNPs in all the studied populations. Significance was calculated using $2 \times 2$ contingency tables and Fisher's exact test or the $\chi^{2}$ test when necessary to obtain $\mathrm{p}$ values, ORs and $95 \% \mathrm{CIs}$ using PLINK (V.1.07) software (http://pngu.mgh.harvard.edu/purcell/ plink/). ${ }^{28}$ The $p$ values less than 0.05 were considered to be statistically significant. The Bonferroni correction was applied to the significant $p$ values and referred in the text as $p_{c}\left(p_{\text {corrected }}\right)$. Cochran-Mantel-Haenszel meta-analysis was performed to control the differences among populations as implemented by the PLINK software. In addition, the Breslow-Day test (BD test) and the Higgins' test $\left(\mathrm{I}^{2}\right)$ were performed using the PLINK software in each meta-analysis. The random-effects model was checked in the significant $\mathrm{BD} \mathrm{P}_{\text {values }}$ analysis. The dependency of the association between each SNP and every studied genetic variant was determined by a conditional logistic regression analysis (considering the different cohorts as covariates) using the PLINK software. Linkage disequilibrium (LD) patterns between the four studied SNPs were estimated by the expectation-maximisation algorithm using HAPLOVIEW (V.4.2; Broad Institute of MIT and Harvard) and PLINK software. To evaluate the allelic combination difference between cases and controls, the conditional haplotype-based associations test was applied using the PLINK software. ${ }^{29}$ The statistical power of the combined analysis was between $91 \%$ and $99 \%$ for all the SNPs, allowing for the detection of associations with an OR equal to 1.2 at a $5 \%$ significance level and the lowest minor allelic frequency, according to the Power Calculator for Genetic Studies 2006 software, which uses the methods described by Skol et al. ${ }^{30}$

\section{RESULTS}

The cases and controls of the eight Caucasian populations were in Hardy-Weinberg equilibrium at a $5 \%$ significance level. Additionally, the minor allelic frequencies of the four studied SNPs were similar to those reported by the HapMap project for the Utah residents with ancestry from northern and western Europe (CEU) population (http://hapmap.ncbi.nlm.nih.gov/). The LD structure of the eight cohorts is shown in the supplemental material (see online supplementary figure S1).

First, an association study was conducted in a Spanish casecontrol set, and a significant association was observed between the rs 907715 SNPs minor allele and the global SSc $\left(p_{c}=0.03\right.$, $\mathrm{OR}=0.8595 \%$ CI 0.8 to 0.9 ) and the lcSSc subtype ( $p_{c}=0.04$, $\mathrm{OR}=0.8395 \%$ CI 0.7 to 0.9 ). A trend of association was observed between the minor allele of the rs6822844 SNP and the global SSc ( $p_{\text {value }}=0.04, \mathrm{OR}=0.8495 \% \mathrm{CI} 0.7$ to 1 ) and lcSSc subtype ( $p_{\text {value }}=0.04, \mathrm{OR}=0.7995 \% \mathrm{CI} 0.7$ to 0.9 ). Also a trend of association was detected between the minor allele of rs6835457 and lcSSc subtype in this population ( $p_{\text {value }}=0.03$, $\mathrm{OR}=0.8795 \%$ CI 0.8 to 1 ). In contrast, no association was observed with the rs2069762 SNP ( $p_{\text {value }}=0.8$ for both SSc and 1cSSc) (see online supplementary tables S1-S3). Based on these observations, we decided to evaluate other Caucasian cohorts and to perform a meta-analysis.

Table 1 shows the meta-analysis results for the $I L-2 / I L-21$ SNPs, the global SSc, the main SSc subtypes, the ACA and the ATA antibodies positive status. The combined analysis showed that the minor allele frequencies of the rs6822844 and rs907715 SNPs were significantly higher in controls than in SSc $\left(p_{c}=6.6 \mathrm{E}-04 \mathrm{OR}=0.8695 \%\right.$ CI 0.79 to 0.93 and $\mathrm{p}_{\mathrm{c}}=7.2 \mathrm{E}-3 \mathrm{OR}=0.9195 \%$ CI 0.85 to 0.96 , respectively) 
Table 1 Genotype and minor allele frequencies of meta-analysis of four IL-2/IL-21 SNPs located in SSc patients and healthy controls from European and US populations

\begin{tabular}{|c|c|c|c|c|c|c|c|c|c|}
\hline \multirow[b]{2}{*}{ SNP } & \multirow[b]{2}{*}{$1 / 2$} & \multirow[b]{2}{*}{ Subgroup (N) } & \multicolumn{3}{|c|}{ Genotype, N (\%) } & \multirow[b]{2}{*}{ MAF (\%) } & \multicolumn{3}{|l|}{ Allele test } \\
\hline & & & $1 / 1$ & $1 / 2$ & $2 / 2$ & & p Value* & $p_{c} \dagger$ & OR (CI 95\%)‡ \\
\hline \multirow[t]{6}{*}{ rs2069762 } & $\mathrm{C} / \mathrm{A}$ & Controls $(n=5482)$ & $510(9.30)$ & 2266 (41.34) & $2706(49.36)$ & 29.97 & & & \\
\hline & & SSc $(n=4281)$ & $429(10.02)$ & $1778(41.53)$ & $2074(48.45)$ & 30.79 & 0.08 & NA & $1.06(0.99$ to 1.13$)$ \\
\hline & & IcSSc $(n=2897)$ & $295(10.18)$ & $1203(41.53)$ & $1399(48.29)$ & 30.95 & 0.09 & NA & $1.06(0.99$ to 1.14$)$ \\
\hline & & dcSSc $(n=1384)$ & $134(9.68)$ & $575(41.55)$ & $675(48.77)$ & 30.46 & 0.31 & NA & $1.05(0.96$ to 1.15$)$ \\
\hline & & $A C A+(n=1736)$ & $170(9.79)$ & $730(42.05)$ & $836(48.16)$ & 30.82 & 0.25 & NA & $1.05(0.97$ to 1.14$)$ \\
\hline & & $A T A+(n=1031)$ & $94(9.12)$ & $428(41.51)$ & 509 (49.37) & 29.87 & 0.98 & NA & $1.00(0.90$ to 1.11$)$ \\
\hline \multirow[t]{6}{*}{ rs6822844 } & $T / G$ & Controls $(n=5792)$ & $149(2.57)$ & $1475(25.47)$ & $4168(71.96)$ & 15.31 & & & \\
\hline & & SSc $(n=4407)^{* *}$ & $98(2.22)$ & $996(22.60)$ & $3313(75.18)$ & 13.52 & $1.7 \mathrm{E}-04$ & $6.6 \mathrm{E}-04$ & $0.86(0.79$ to 0.93$)$ \\
\hline & & $\operatorname{lcSSc}(n=2977)^{* * *}$ & $67(2.25)$ & $659(22.14)$ & $2251(75.61)$ & 13.32 & $1.5 \mathrm{E}-04$ & $6.0 \mathrm{E}-04$ & $0.84(0.76$ to 0.92$)$ \\
\hline & & dcSSc $(n=1430)$ & $31(2.17)$ & 337 (23.57) & 1062 (74.27) & 13.95 & 0.06 & NA & $0.89(0.79$ to 1$)$ \\
\hline & & $A C A+(n=1763)$ & $38(2.16)$ & $395(22.40)$ & $1330(75.44)$ & 13.36 & 0.01 & 0.06 & 0.87 (0.78 to 0.97$)$ \\
\hline & & $A T A+(n=1074)$ & $29(2.70)$ & $257(23.93)$ & $788(73.37)$ & 14.66 & 0.67 & NA & $0.97(0.85$ to 1.11$)$ \\
\hline \multirow[t]{6}{*}{ rs6835457 } & $\mathrm{G} / \mathrm{A}$ & Controls $(n=5720)$ & $668(11.68)$ & $2507(43.83)$ & $2545(44.49)$ & 33.59 & & & \\
\hline & & SSc $(n=4392)^{* * * *}$ & $445(10.13)$ & $1908(43.44)$ & $2039(46.43)$ & 31.85 & 0.013 & 0.05 & $0.93(0.87$ to 0.98$)$ \\
\hline & & $\operatorname{lcSSc}(n=2965)^{* * * * *}$ & $312(10.52)$ & $1255(42.33)$ & $1398(47.15)$ & 31.69 & 0.014 & 0.06 & $0.92(0.86$ to 0.98$)$ \\
\hline & & dcSSc $(n=1427)$ & $133(9.32)$ & $653(45.76)$ & $641(44.92)$ & 32.20 & 0.28 & NA & $0.95(0.87$ to 1.04$)$ \\
\hline & & $A C A+(n=1765)$ & $186(10.54)$ & $756(42.83)$ & $823(46.63)$ & 31.95 & 0.12 & NA & $0.94(0.86$ to 1.02$)$ \\
\hline & & ATA $+(n=1064)$ & $113(10.62)$ & $481(45.21)$ & $470(44.17)$ & 33.22 & 0.99 & NA & $1.00(0.90$ to 1.10$)$ \\
\hline \multirow[t]{6}{*}{ rs907715 } & $\mathrm{T} / \mathrm{C}$ & Controls $(n=5644)$ & $670(11.87)$ & 2491 (44.14) & $2483(43.99)$ & 33.94 & & & \\
\hline & & $\operatorname{SSc}(n=4341)^{* * * * * *}$ & 437 (10.07) & $1883(43.38)$ & $2021(46.56)$ & 31.76 & $1.8 \mathrm{E}-03$ & 7.2E-03 & $0.91(0.85$ to 0.96$)$ \\
\hline & & $\operatorname{lcSSc}(n=2929) * * * * * *$ & 307 (10.48) & $1236(42.20)$ & $1386(47.32)$ & 31.58 & 2.7E-03 & 0.01 & $0.90(0.84$ to 0.96$)$ \\
\hline & & $\mathrm{dcSSc}(\mathrm{n}=1412)$ & $130(9.21)$ & $647(45.82)$ & $635(44.97)$ & 32.12 & 0.14 & NA & $0.93(0.85$ to 1.02$)$ \\
\hline & & $A C A+(n=1744)$ & $180(10.32)$ & $754(43.23)$ & $810(46.44)$ & 31.94 & 0.05 & NA & $0.92(0.85$ to 1$)$ \\
\hline & & $A T A+(n=1056)$ & 109 (10.32) & 475 (44.98) & $472(44.70)$ & 32.81 & 0.48 & NA & $0.96(0.87$ to 1.07$)$ \\
\hline
\end{tabular}

\footnotetext{
${ }^{*}$ All $p$ values have been calculated for the allelic model.

**Beslow-Day $p_{\text {value }}=0.29$. Higgins' test $\left(\mathrm{I}^{2}\right)=17.3 \%$. Random-effects model $\mathrm{p}_{\text {value }}=8.8 \mathrm{E}-04 \mathrm{p}_{\mathrm{c}}=3.5 \mathrm{E}-3$ Random-effects $0 \mathrm{R}=0.86$.

${ }^{* *}$ Breslow-Day $\mathrm{p}_{\text {value }}=0.16 . \mathrm{I}^{2}=33.9 \%$. Random-effects model $\mathrm{p}_{\text {value }}=4.1 \mathrm{E}-03$. Random-effects $\mathrm{OR}=0.84$.

****Beslow-Day $p_{\text {value }}=0.06 . \mathrm{I}^{2}=48.6 \%$. Random-effects model $\mathrm{p}_{\mathrm{value}}=0.1$. Random-effects OR estimate $=0.93$.

$* * * *$ Breslow-Day $p_{\text {value }}=0.09 . I^{2}=43.4 \%$. Random-effects model $p_{\text {value }}=0.11$. Random-effects 0 R estimate $=0.92$.

$* * * * *$ Breslow-Day $p_{\text {value }}=0.02 .1^{2}=58 \%$. Random-effects model $p_{\text {value }}=0.08$. Random-effects 0 estimate $=0.91$.

$* * * * * *$ Breslow-Day $p_{\text {value }}=0.09 . I^{2}=43.7 \%$. Random-effects model $p_{\text {value }}=0.05$. Random-effects 0R estimate $=0.91$.

tff it is applicable, Bonferroni correction is shown.

$\ddagger 0 \mathrm{R}$ for the minor allele.

ACA, anticentromere autoantibodies; ATA, antitopoisomerase autoantibodies; dcSSc, diffuse cutaneous SSc; NA, not applicable; SNP, single nucleotide polymorphisms;

SSc, systemic sclerosis.
}

and $\mathrm{lcSSc}$ patients $\left(\mathrm{p}_{\mathrm{c}}=6 \mathrm{E}-4 \mathrm{OR}=0.8495 \% \mathrm{CI} 0.76\right.$ to 0.92 and $\mathrm{p}_{\mathrm{c}}=0.01 \mathrm{OR}=0.995 \% \mathrm{CI} 0.84$ to 0.96 , respectively). A trend of association was observed in the meta-analysis for the rs6822844 and rs6835457 variants and ACA positive status $\left(p_{\text {value }}=0.01 \quad O R=0.87 \quad 95 \%\right.$ CI 0.78 to 0.97 and $\mathrm{p}_{\text {value }}=0.05 \mathrm{OR}=0.9295 \% \mathrm{CI} 0.85$ to 1 , respectively). The rs6835457 SNP also had a trend of association with global

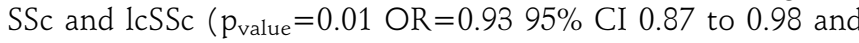

Table 2 Conditional logistic regression analysis for the IL-2/IL-21 SNPs located in SSc considering the eight European and US populations as covariate

\begin{tabular}{|c|c|c|c|c|c|c|c|c|c|c|c|c|c|}
\hline \multirow[b]{2}{*}{$\begin{array}{l}\text { Group of } \\
\text { analysis }\end{array}$} & \multirow[b]{2}{*}{ SNP } & \multirow[b]{2}{*}{$\begin{array}{l}\text { MAF } \\
\text { Cases }\end{array}$} & \multirow[b]{2}{*}{$\begin{array}{l}\text { MAF } \\
\text { Controls }\end{array}$} & \multirow[b]{2}{*}{$\begin{array}{l}\text { p value of each SNP } \\
\text { conditioned by rs } 6822844\end{array}$} & \multirow[b]{2}{*}{$\begin{array}{l}\text { p value of rs } 6822844 \\
\text { conditioned by each SNP }\end{array}$} & \multicolumn{8}{|c|}{ r2 with rs 6822844} \\
\hline & & & & & & Spain & Germany & $\begin{array}{l}\text { The } \\
\text { Netherlands }\end{array}$ & USA & Italy & Sweden & UK & Norway \\
\hline \multicolumn{14}{|l|}{ SSc } \\
\hline & rs2069762 & 0.31 & 0.30 & 0.69 & $1.30 \mathrm{E}-03$ & 0.06 & 0.06 & 0.09 & 0.07 & 0.07 & 0.11 & 0.06 & 0.09 \\
\hline & rs6835457 & 0.32 & 0.34 & 0.43 & 0.024 & 0.25 & 0.37 & 0.38 & 0.36 & 0.28 & 0.48 & 0.37 & 0.49 \\
\hline & rs907715 & 0.32 & 0.34 & 0.19 & 0.026 & 0.26 & 0.37 & 0.39 & 0.36 & 0.29 & 0.39 & 0.37 & 0.49 \\
\hline \multicolumn{14}{|l|}{ IcSSc } \\
\hline & rs2069762 & 0.31 & 0.30 & 0.69 & 9.07E-04 & - & - & - & - & - & - & - & - \\
\hline & rs6835457 & 0.32 & 0.34 & 0.53 & 0.014 & - & - & - & - & - & - & - & - \\
\hline & rs907715 & 0.32 & 0.34 & 0.3 & 0.015 & - & - & - & - & - & - & - & - \\
\hline \multicolumn{14}{|l|}{$\mathrm{ACA}+$} \\
\hline & rs2069762 & 0.31 & 0.30 & 0.64 & 0.015 & - & - & - & - & - & - & - & - \\
\hline & rs6835457 & 0.32 & 0.34 & 0.81 & 0.061 & - & - & - & - & - & - & - & - \\
\hline & rs 907715 & 0.32 & 0.34 & 0.56 & 0.063 & - & - & - & - & - & - & - & - \\
\hline
\end{tabular}

ACA, anticentromere autoantibodies; IcSSC, limited cutaneous SSc; MAF, minor allelic frequencies; SNP, single nucleotide polymorphisms; SSc, systemic sclerosis. 
$p_{\text {value }}=0.01 \mathrm{OR}=0.9295 \% \mathrm{CI} 0.86$ to 0.98 , respectively). We did not detect any significant association between the rs6835457 or rs2069762 SNPs and the global SSc diseases or its different phenotypes (for detailed information see supplementary tables S1 through S3). It is worth noting that the minor $\mathrm{I}^{2}$ percentage was observed in the meta-analysis for the rs6822844 SNP with SSc (17.3\%) and 1cSSc (33.9\%), suggesting that the variation between the populations is moderate. Moreover, these analyses were the only ones that remained significant in the random-effect model (rs6822844 and SSc $\mathrm{p}_{\mathrm{c}}=3.5 \mathrm{E}-3$, rs6822844 and $1 \mathrm{cSSc} \mathrm{p}_{\mathrm{c}}=0.016$ ).

A conditional logistic regression analysis was used to identify which SNP could be the causal SNP for the observed associations between the studied polymorphisms. The association of each SNP was evaluated using the populations as covariates, and the association was conditioned to the rs6822844 SNP because the lowest $p_{\text {value }}$ and strongest effect (OR) were observed in this locus. Pairwise conditional analysis showed that the association of the rs907715 SNP was explained by the rs6822844 effect, because only the latter SNP remained significant after conditioned to each other (rs907715 conditioned $p_{\text {value }}=0.19 ;$ rs6822844 conditioned $p_{\text {value }}=0.026$ ). Moreover, the rs2069762 and rs6835457 SNPs exhibited significance only when conditioned to the rs6822844 SNP. These results suggested that the rs6822844 signal could explain the association observed in the $I L-2 / I L-21$ locus (table 2 ).

Finally, the results of the conditional haplotype-based association testing are shown in table 3 . The allelic combination formed by the rs2069762 major allele and the rs6822844, rs6835457 and rs907715 minor alleles was significantly increased in the controls compared with the global SSc $\left(p_{c}=1.7 \mathrm{E}-3, \mathrm{OR}=0.8995 \% \mathrm{CI} 0.81\right.$ to 0.98$)$, the lcSSc subtype $\left(p_{c}=8 E-4, O R=0.8695 \%\right.$ CI 0.77 to 0.96$)$ and the ACA positive status $\quad\left(p_{c}=2.7 \mathrm{E}-2, \quad O R=0.86 \quad 95 \% \quad C I \quad 0.75\right.$ to 0.98$)$. Interestingly, the OR observed for this analysis was not different from the one observed in the allelic test. Moreover, the significant effect of the omnibus analyses for SSc, IcSSc and ACA positive status disappeared when they were controlled by the rs6822844 SNP ( $p_{\text {values }}$ of the likelihood ratio test were: $p_{\text {value }}=0.66$ for global SSc, $p_{\text {value }}=0.74$ for $1 c S S c$ and $p_{\text {value }}=0.93$ for $\mathrm{ACA}+$ ).

\section{DISCUSSION}

Our study suggests for the first time the influence of the rs6822844 polymorphism of the IL-2/IL-21 region in susceptibility to SSc. This variant also influences the lcSSc subtype of the diseases and probably the ACA positive status due to the trend of association observed between the rs6822844 polymorphism and this phenotype. Although, our study had sufficient statistical power for both dcSSc and ATA analysis $(95 \%$ and $91 \%$, respectively), we observed that there were not significant associations between the four $I L-2 / I L-21$ SNPs and dcSSc or ATA positive status. The ORs exhibited the same direction as the significant associations with $\mathrm{SSc}$ and $\mathrm{lcSSc}$, suggesting that an increment in the sample size with future studies could show a significant relation between the IL-2/IL-21 SNPs and $\mathrm{dcSSc}$ or ATA. Interestingly, the rs6822844 variant was associated in the same OR direction as that observed in SLE. The minor allele of this variant is more frequent in healthy donors than in SSc patients, lcSSc subtype subjects and SLE patients. ${ }^{12} 16$ The logistic regression and the allelic combination analyses support that the rs6822844 SNP association was responsible for the observed associations. The rs2069762Ars6822844T-rs6835457G-rs907715T allelic combination was associated as a protective factor to SSc, lcSSc subtype and ACA positive status, which is the same effect observed for the $T$ allele of the rs6822844. Importantly, the ORs observed for this allelic combination were not different from the ORs observed for the rs6822844 SNP analysis. These observations were slightly different from the results of the SLE study performed by Hughes et al ${ }^{12}$ where the observed association between IL-2/IL-21 region and SLE could be explained by the rs6835457 and rs907715 SNPs. Together, these results support the idea that the common genetic factors in autoimmune diseases may be associated at a regional level but differ in the specific SNPs associated with each disease, including the magnitude and direction of the association. ${ }^{31}{ }^{32}$ Although, the logistic regression test and the allelic combination analyses conditioned by the rs6822844 SNP suggest that this variant is responsible for the association observed in the region; we cannot totally discard a slight role of the rs6835457 and rs907715 polymorphisms in SSc due to the moderate $\mathrm{LD}$ between them and the r6822844 SNP.

The rs6822844 and rs6835457 SNPs are located in the flanking 3 '-untranslated region of $I L-21$, and the rs 907715 polymorphism is located in intron 3 of the $I L-21$ gene. In contrast, the rs2069762 SNP is located in the flanking 5'-untranslated region of $I L-2$, which did not exhibit significant association with SSc, the subtypes of the disease or the antibodies' status. The rs2069762 minor allele has been previously associated with the lcSSc subtype. ${ }^{26}$ Our study has a considerably larger sample size than the previous study; therefore the previously reported

Table 3 Conditional haplotype-based association analysis of four IL-2/IL-21 SNPs located according to diseases, IcSSc diseases subtype and ACA status and considering the eight European and US populations as covariate

\begin{tabular}{|c|c|c|c|c|c|c|c|c|c|c|c|c|c|}
\hline $\begin{array}{l}\text { Allelic } \\
\text { combinationt }\end{array}$ & \multicolumn{5}{|c|}{ Frequency } & \multicolumn{4}{|c|}{ Frequency } & \multicolumn{4}{|c|}{ Frequency } \\
\hline ATGT & 0.152 & 0.133 & 0.89 (0.81 to 0.98 ) & $4.12 \mathrm{E}-04$ & $1.65 \mathrm{E}-03$ & 0.131 & 0.86 (0.77 to 0.96$)$ & 2.01E-04 & 8.04E-04 & 0.132 & 0.86 (0.75 to 0.98 ) & $6.81 \mathrm{E}-03$ & $2.72 \mathrm{E}-02$ \\
\hline CGAC & 0.298 & 0.306 & 1.04 (0.96 to 1.12 ) & 0.18 & NA & 0.308 & $1.03(0.94$ to 1.13$)$ & 0.2 & NA & 0.308 & 1.02 (0.92 to 1.14$)$ & 0.27 & NA \\
\hline AGAC & 0.369 & 0.379 & 1.04 (0.96 to 1.13 ) & 0.1 & NA & 0.38 & $1.03(0.94$ to 1.13$)$ & 0.12 & NA & 0.377 & $1.02(0.91$ to 1.13$)$ & 0.4 & NA \\
\hline
\end{tabular}

tThe order of the SNPs is rs2069762, rs6822844, rs6835457, rs907715.

${ }^{*} p_{\text {value }}$ of the likelihood ratio test. Based on WHAP method. ${ }^{29}$

flf it is applicable, Bonferroni correction is shown. Not applicable (NA).

**0mnibus test $X^{2}=14.5(\mathrm{df}=3) ; \mathrm{p}$ value $=2.35 \mathrm{E}-03 ; \mathrm{Pc}=9.4 \mathrm{E}-03$.

$* * * 0$ mnibus test $X^{2}=14.5(\mathrm{df}=3) ; p$ value $=2.32 \mathrm{E}-03 ; \mathrm{Pc}=9.28 \mathrm{E}-03$.

$* * * *$ mnibus test $X^{2}=8.92(\mathrm{df}=3) ; p$ value $=0.03 ; \mathrm{Pc}=0.12$.

ACA, anticentromere autoantibodies; IcSSc, limited cutaneous SSc; SNP, single nucleotide polymorphisms; SSc, systemic sclerosis. 
significant association for rs2069762 might stem from type 1 statistical error. This fact together with the location of the associated SNP suggests a highlighted role of the IL-21 cytokine. By examining the expression and regulation of $I L-21$ and the $I L-21$ receptor $(I L-21 R)$ in patients with SSc, a previous study demonstrated an upregulation of $I L-21 R$ in epidermis samples. ${ }^{33}$ However, a recent study has demonstrated that the scleroderma burden in allogeneic haemopoietic stem cell transplantations is driven by Th17 induction via $I L-21$ and $I L-23$ signalling. ${ }^{34}$ Together, these results suggest that $I L-21 / I L-21 R$ signalling has a pathogenic function in SSc.

The role of $I L-2$ and $I L-21$ in the immune system makes these genes plausible candidates for the genetic component of autoimmune diseases. ${ }^{13536}$ Our results increase the evidence that have showed that the rs6822844 is significantly associated with multiple autoimmune diseases. ${ }^{10-13}$ According to the HapMap project for the CEU population (http://hapmap.ncbi. nlm.nih.gov/), the rs682284 polymorphism tags seven other variants located along the $I L-2 / I L-21$ region (rs13132245, rs13122573, rs4459999, rs13151961, rs13140464, rs6814280 and rs2069778), but clear evidence that connects any of these variants with the $I L-2$ and/or $I L-21$ regulation is lacking. Together, all point out these genetic variants as good candidates for functional studies in SSc pathogenesis and in other autoimmune diseases.

Although, the combined analyses of the rs6822844 polymorphism did not show heterogeneity (BD $p_{\text {value }}=0.29$. $\mathrm{I}^{2}=17.3 \%$ ) between the eight European populations, a weak point of our study is that we did not have enough data available to control the association by principal component. Furthermore, as we mentioned before, an increment in the sample size for the stratified analysis could define in an accurate way the role of the studied variants in different clinical manifestations of $\mathrm{SSc}$ as their influence in the presence of coautoimmunity. Consequently, it is necessary to replicate the actual observation.

To conclude, consistent with previous studies on autoimmune diseases, the IL-2/IL-21 region is a susceptibility genetic factor for SSc and its lcSSc subtype. The rs6822844 polymorphism confers the best association signal for SSc. It is also worth mentioning that this study shows the importance of the study of different populations and broad collaboration to find the missing heritability for relatively rare diseases like SSc.

\footnotetext{
Author affiliations

${ }^{1}$ Cellular Biology and Immunology Department, Instituto de Parasitología y Biomedicina López-Neyra, IPBLN-CSIC, Granada, Spain

${ }^{2}$ Department of Internal Medicine, Hospital Vall d'Hebron, Barcelona, Spain ${ }^{3}$ Department of Rheumatology, Radboud University Nijmegen Medical Centre, Nijmegen, The Netherlands

${ }^{4}$ Department of Internal Medicine, Hospital Clínico Universitario San Cecilio, Granada, Spain

${ }^{5}$ Department of Allergy, Clinical Immunology and Rheumatology, IRCCS Fondazione Policlinico-Mangiagalli-Regina Elena \& University of Milan, Milan, Italy

${ }^{6}$ Department of Rheumatology, Hospital Universitario 12 de Octubre, Madrid, Spain

${ }^{7}$ Department of Rheumatology, Hospital Universitario y Politécnico La Fe, Valencia, Spain

${ }^{8}$ Department of Rheumatology, Hospital Marqués de Valdecilla, IFIMAV, Santander, Spain

${ }^{9}$ Department of Internal Medicine, Hospital Parc Tauli, Sabadell, Spain

${ }^{10}$ Department of Rheumatology, Hospital General Universitario Gregorio Marañon, Madrid, Spain

${ }^{11}$ Department of Systemic Autoimmune Diseases, Hospital Clinic de Barcelona, Barcelona, Spain

${ }^{12}$ Department of Rheumatology, Hospital Universitario La Princesa, Madrid, Spain

${ }^{13}$ Department of Rheumatology, Lund University Hospital, Lund, Sweden

${ }^{14}$ Department of Rheumatology and Clinical Immunology, Charité Universitátsmedizin and German Rheumatism Research Centre, a Leibniz institute. Berlin, Germany
}

${ }^{15}$ Department of Clinical Immunology and Rheumatology, Hannover Medical School, Hannover, Germany

${ }^{16}$ Department of Internal Medicine 3, Institute for Clinical Immunology, University of

Erlangen-Nuremberg, Erlangen, Germany

${ }^{17}$ Department of Rheumatology, VU University Medical Center, Amsterdam,

The Netherlands

${ }^{18}$ Department of Rheumatology, Leiden University Medical Center, Leiden,

The Netherlands

${ }^{19}$ Institute of Cancer Sciences, College of Medical, Veterinary and Life Sciences, University of Glasgow, Glasgow, Scotland

${ }^{20}$ Rheumatology Unit, Department of Medicine, Karolinska Institutet, Stockholm, Sweden

${ }^{21}$ Department of Rheumatology, Rikshospitalet, Oslo University Hospital, Oslo, Norway

${ }^{22}$ Department of Medicine, Università degli Studi di Verona, Verona, Italy

${ }^{23} \mathrm{UO}$ Reumatologia ed Immunologia Clinica, Spedali Civili, Brescia, Italy

${ }^{24}$ Musculoskeletal Research Group, Institute of Cellular Medicine, Newcastle

University, Newcastle, UK

${ }^{25}$ Department of Dermatology, University of Cologne, Cologne, Germany

${ }^{26}$ Division of Transfusion Medicine, University Hospital of Cologne, Cologne, Germany

${ }^{27}$ Department of Dermatology, Allergology, and Venereology, Ruhr University of

Bochum, Bochum, Germany

${ }^{28}$ Arthritis Research UK Epidemiology Unit, The University of Manchester, Manchester Academic Health Science Centre, Manchester, UK

${ }^{29}$ Centre for Rheumatology, Royal Free and University College Medical School, London, UK

${ }^{30}$ Department of Rheumatology and Clinical Immunogenetics, The University of Texas Health Science Center at Houston, Houston, Texas, USA

${ }^{31}$ Section Complex Genetics, Department of Medical Genetics, University Medical Center Utrecht, Utrecht, The Netherlands

${ }^{32}$ Department of Rheumatology \& Clinical Immunology, University Medical Center Utrecht, Utrecht, The Netherlands

Acknowledgements We thank Sonia García and Gema Robledo for their excellent technical assistance and all the patients and control donors for their essential collaboration. We thank Banco Nacional de ADN (University of Salamanca, Spain). We are also thankful to EUSTAR (The EULAR Scleroderma Trials and Research group) and the German Network of Systemic Sclerosis for the facilitation of this project.

Spanish Scleroderma Group Jose Luis Callejas and Raquel Ríos, Unidad de Enfermedades Sistémicas Autoinmunes, Servicio de Medicina Interna, Hospital Clínico Universitario San Cecilio, Granada; Nuria Navarrete, Servicio de Medicina Interna, Hospital Virgen de las Nieves, Granada; Rosa García Portales, Servicio de Reumatología, Hospital Virgen de la Victoria, Málaga; María Teresa Camps, Servicio de Medicina Interna, Hospital Carlos Haya, Málaga; Antonio Fernández-Nebro, Servicio de Reumatología, Hospital Carlos Haya, Málaga; María F. González-Escribano, Servicio de Inmunología, Hospital Virgen del Rocío, Sevilla; Julio Sánchez-Román, Francisco J García-Hernández and Ma Jesús Castillo, Servicio de Medicina Interna, Hospital Virgen del Rocío, Sevilla; Ma Ángeles Aguirre and Inmaculada Gómez-Gracia, Servicio de Reumatología, Hospital Reina Sofía, Córdoba; Benjamín Fernández-Gutiérrez and Luis Rodríguez-Rodríguez, Servicio de Reumatología, Hospital Clínico San Carlos, Madrid; José Luis Andreu, Servicio de Reumatología, Hospital Puerta del Hierro, Madrid; Paloma García de la Peña, Servicio de Reumatología, Hospital Madrid Norte Sanchinarro, Madrid; Lina Martínez, Servicio de Reumatología, Hospital General Universitario Gregorio Marañón, Madrid; María Ángeles Robles, Natividad Oreiro, Servicio de Reumatología, INIBIC-Hospital Universitario A Coruña, La Coruña; Vicente Fonollosa, Servicio de Medicina Interna, Hospital Valle de Hebrón, Barcelona; Anna Pros, Servicio de Reumatología, Hospital Del Mar, Barcelona; Mónica Rodríguez Carballeira, Servicio de Medicina Interna, Hospital Universitari Mútua Terrasa, Barcelona; Francisco Javier Narváez, Servicio de Reumatología, Hospital Universitari de Bellvitge, Barcelona; Bernardino Díaz, Luis Trapiella and María Gallego, Servicio de Medicina Interna, Hospital Central de Asturias, Oviedo; María del Carmen Freire and Inés Vaqueiro, Unidad de Trombosis y Vasculitis, Servicio de Medicina Interna, Hospital Xeral-Complexo Hospitalario Universitario de Vigo, Vigo; María Victoria Egurbide, Servicio de Medicina Interna, Hospital de Cruces, Barakaldo; Luis Sáez-Comet, Unidad de Enfermedades Autoinmunes Sistémicas, Servicio de Medicina Interna, Hospital Universitario Miguel Servet, Zaragoza; Federico Díaz and Vanesa Hernández, Servicio de Reumatología, Hospital Universitario de Canarias, Tenerife; Emma Beltrán, Hospital General Universitario, Valencia Spain.

Contributors All the authors listed participated in all or at list one of these activities: Conception and design, acquisition of data or analysis and interpretation of data. Drafting the article or revising it critically for important intellectual content. Final approval of the version published.

Funding This work was supported by the following grants: LMDG was funded by the 'Ayudas Predoctorales de Formación en Investigación en Salud (PFIS-FI09/00544)' from the Instituto de Salud Carlos III. JM was funded by GEN-FER from the Spanish 
Society of Rheumatology, SAF2009-11110 from the Spanish Ministry of Science, CTS-4977 from Junta de Andalucía, Spain, in part by Redes Temáticas de Investigación Cooperativa Sanitaria Program, RD08/0075 (RIER) from Instituto de Salud Carlos III (ISCIII), Spain and by Fondo Europeo de Desarrollo Regional (FEDER). TRDJR was funded by the VIDI laureate from the Dutch Association of Research (NWO) and Dutch Arthritis Foundation (National Reumafonds). JM and TRDJR were sponsored by the Orphan Disease Program grant from the European League Against Rheumatism (EULAR). BPCK is supported by the Dutch Diabetes Research Foundation (grant 2008.40.001) and the Dutch Arthritis Foundation (Reumafonds, grant NR 09-1-408). TW was granted by DFG WI 1031/6.1 and DFG KFO 250 TP03. NOC was funded by PI-0590-2010, Consejería de Salud, Junta de Andalucía, Spain.US National Institutes of Health and National Institute of Arthritis and Musculoskeletal Diseases (NIH-NIAMS) R01-AR-055258, Two-Stage Genome Wide Association Study in Systemic Sclerosis and by the NIH-NIAMS Center of Research Translation (CORT) in SSc (P50AR054144), K23AR061436, and the Department of Defense Congressionally Directed Medical Research Programs (W81XWH-07-01-0111), (NIH/NIAID) 1 U01Al09090.

Competing interests None.

Ethics approval The local ethical committees in accordance with the tenets of the Declaration of Helsinki.

Provenance and peer review Not commissioned; externally peer reviewed.

\section{REFERENCES}

1. McGuire HM, Vogelzang A, Hill N, et al. Loss of parity between IL-2 and IL-21 in the NOD Idd3 locus. Proc Natl Acad Sci USA 2009;106:19438-43.

2. Yamanouchi J, Rainbow D, Serra $P$, et al. Interleukin-2 gene variation impairs regulatory T cell function and causes autoimmunity. Nat Genet 2007;39:329-37.

3. Bubier JA, Sproule TJ, Foreman 0, et al. A critical role for IL-21 receptor signaling in the pathogenesis of systemic lupus erythematosus in BXSB-Yaa mice. Proc Natl Acad Sci USA 2009;106:1518-23.

4. Wellcome Trust Case Control Consortium. Genome-wide association study of 14,000 cases of seven common diseases and 3,000 shared controls. Nature 2007:447:661-78.

5. Todd JA, Walker NM, Cooper JD, et al. Robust associations of four new chromosome regions from genome-wide analyses of type 1 diabetes. Nat Genet 2007;39:857-64.

6. van Heel DA, Franke L, Hunt KA, et al. A genome-wide association study for celiac disease identifies risk variants in the region harboring IL2 and IL21. Nat Genet 2007:39:827-9.

7. Zhernakova A, Alizadeh BZ, Bevova M, et al. Novel association in chromosome 4q27 region with rheumatoid arthritis and confirmation of type 1 diabetes point to a general risk locus for autoimmune diseases. Am J Hum Genet 2007;81:1284-8.

8. Barton A, Eyre $S, K e X$, et al. Identification of AF4/FMR2 family, member 3 (AFF3) as a novel rheumatoid arthritis susceptibility locus and confirmation of two further pan-autoimmune susceptibility genes. Hum Mol Genet 2009;18:2518-22.

9. Festen EA, Goyette P, Scott R, et al. Genetic variants in the region harbouring IL2/IL21 associated with ulcerative colitis. Gut 2009;58:799-804.

10. Hollis-Moffatt JE, Chen-Xu M, Topless R, et al. Only one independent genetic association with rheumatoid arthritis within the KIAA1109-TENR-IL2-IL21 locus in Caucasian sample sets: confirmation of association of rs6822844 with rheumatoid arthritis at a genome-wide level of significance. Arthritis Res Ther 2010;12:R116.

11. Hollis-Moffatt JE, Gearry RB, Barclay ML, et al. Consolidation of evidence for association of the KIAA1109-TENR-IL2-IL21 rs6822844 variant with Crohn's disease. Am J Gastroenterol 2010;105:1204-5.

12. Hughes T, Kim-Howard X, Kelly JA, et al. Fine-mapping and transethnic genotyping establish IL2/IL21 genetic association with lupus and localize this genetic effect to IL21. Arthritis Rheum 2011;63:1689-97.
13. Maiti AK, Kim-Howard X, Viswanathan $P$, et al. Confirmation of an association between rs6822844 at the II2-II21 region and multiple autoimmune diseases: evidence of a general susceptibility locus. Arthritis Rheum 2010;62:323-9.

14. Marquez A, Orozco G, Martinez A, et al. Novel association of the interleukin 2-interleukin 21 region with inflammatory bowel disease. Am J Gastroenterol 2009; 104:1968-75

15. Rodriguez-Rodriguez L, Castaneda S, Vazquez-Rodriguez TR, et al. Role of the rs6822844 gene polymorphism at the IL2-IL21 region in biopsy-proven giant cell arteritis. Clin Exp Rheumatol 2011;29(1 Suppl 64):S12-16.

16. Sawalha $\mathbf{A H}$, Kaufman KM, Kelly JA, et al. Genetic association of interleukin-21 polymorphisms with systemic lupus erythematosus. Ann Rheum Dis 2008;67:458-61.

17. Warren RB, Smith RL, Flynn E, et al. A systematic investigation of confirmed autoimmune loci in early-onset psoriasis reveals an association with IL2/L21. Br J Dermatol 2011;164:660-4.

18. Gabrielli A, Avvedimento EV, Krieg T. Scleroderma. N Engl J Med 2009;360:1989-2003

19. Katsumoto TR, Whitfield ML, Connolly MK. The pathogenesis of systemic sclerosis. Annu Rev Pathol 2011;6:509-37.

20. Koenig M, Dieude M, Senecal JL. Predictive value of antinuclear autoantibodies: the lessons of the systemic sclerosis autoantibodies. Autoimmun Rev 2008;7:588-93.

21. LeRoy EC, Black C, Fleischmajer R, et al. Scleroderma (systemic sclerosis): classification, subsets and pathogenesis. J Rheumatol 1988;15:202-5.

22. Martin JE, Bossini-Castillo L, Martin J. Unraveling the genetic component of systemic sclerosis. Hum Genet 2012;131:1023-37.

23. Assassi S, Mayes MD, Arnett FC, et al. Systemic sclerosis and lupus: points in an interferon-mediated continuum. Arthritis Rheum 2010;62:589-98.

24. Carmona FD, Gutala R, Simeon CP, et al. Novel identification of the IRF7 region as an anticentromere autoantibody propensity locus in systemic sclerosis. Ann Rheum Dis 2011;71:114-19.

25. Radstake TR, Gorlova 0, Rueda B, et al. Genome-wide association study of systemic sclerosis identifies CD247 as a new susceptibility locus. Nat Genet 2010;42:426-9.

26. Mattuzzi S, Barbi S, Carletto A, et al. Association of polymorphisms in the IL1B and IL2 genes with susceptibility and severity of systemic sclerosis. J Rheumatol 2007; 34:997-1004

27. Preliminary criteria for the classification of systemic sclerosis (scleroderma). Subcommittee for scleroderma criteria of the American Rheumatism Association Diagnostic and Therapeutic Criteria Committee. Arthritis Rheum 1980:23:581-90.

28. Purcell S, Neale B, Todd-Brown K, et al. PLINK: a tool set for whole-genome association and population-based linkage analyses. Am J Hum Genet 2007:81:559-75

29. Purcell S, Daly MJ, Sham PC. WHAP: haplotype-based association analysis. Bioinformatics 2007:23:255-6.

30. Skol AD, Scott LJ, Abecasis GR, et al. Joint analysis is more efficient than replication-based analysis for two-stage genome-wide association studies. Nat Genet 2006;38:209-13.

31. Cho $\mathbf{J H}$, Gregersen PK. Genomics and the multifactorial nature of human autoimmune disease. N Engl J Med 2011;365:1612-23.

32. Sirota M, Schaub MA, Batzoglou S, et al. Autoimmune disease classification by inverse association with SNP alleles. PLoS Genet 2009;5:e1000792.

33. Distler JH, Jungel A, Kowal-Bielecka 0, et al. Expression of interleukin-21 receptor in epidermis from patients with systemic sclerosis. Arthritis Rheum 2005; 52:856-64

34. Hill GR, Olver SD, Kuns RD, et al. Stem cell mobilization with G-CSF induces type 17 differentiation and promotes scleroderma. Blood 2010;116:819-28.

35. Crispin JC, Tsokos GC. Transcriptional regulation of IL-2 in health and autoimmunity. Autoimmun Rev 2009;8:190-5.

36. Ettinger R, Kuchen S, Lipsky PE. Interleukin 21 as a target of intervention in autoimmune disease. Ann Rheum Dis 2008;67(Suppl 3):iii83-6. 